\title{
A multi-institutional feasibility study of S-1/oxaliplatin plus bevacizumab in patients with advanced/metastatic colorectal cancer: the HiSCO-02 prospective phase II study
}

Manabu Shimomura', Katsunori Shinozaki ${ }^{2}$, Takao Hinoi ${ }^{1}$, Masanori Yoshimitsu ${ }^{3}$, Manabu Kurayoshi ${ }^{4}$, Daisuke Sumitani ${ }^{5}$, Yasuyo Ishizaki ${ }^{6}$, Takafumi Oshiro ${ }^{7}$, Shinya Kodama ${ }^{8}$, Yosuke Shimizu ${ }^{9}$, Michinori Arita $^{10}$, Masakazu Tokunaga ${ }^{11}$, Makoto Yoshida ${ }^{12}$, Junko Tanaka ${ }^{13}$, Hideki Ohdan ${ }^{1}$ and Hiroshima Surgical study group of Clinical Oncology (HiSCO)

\begin{abstract}
Purpose: FOLFOX is a standard combination chemotherapy regimen for metastatic colorectal cancer (CRC). 5-Fluorouracil (5-FU) is infused continuously through a pump for 46 h; therefore, replacement of infused 5-FU with oral S-1 would be more convenient for patients. We investigated the efficacy and safety of S-1/oxaliplatin (SOX) plus bevacizumab regimen in a community setting.
\end{abstract}

Methods: We conducted a phase II clinical study in Hiroshima, Japan. We enrolled individuals aged 20-80 years who had metastatic CRC, an Eastern Cooperative Oncology Group performance status of 0 or 1, assessable lesions, and not received previous chemotherapy. Eligible patients were administered SOX plus bevacizumab (S-1 $80 \mathrm{mg} / \mathrm{m}^{2} /$ day, day 1-14 orally; and oxaliplatin $130 \mathrm{mg} / \mathrm{m}^{2}$ day 1 i.v., bevacizumab $7.5 \mathrm{mg} / \mathrm{kg}$, day 1 i.v. q3w). The primary endpoint was response rate (RR), and the secondary endpoints were progression-free survival (PFS), overall survival (OS), and safety.

Results: Between May 2011 and January 2014, 55 patients (mean age 64 years) were enrolled at 12 institutions. Median follow up duration was 20.2 months (range 1.3-47.1 months). RR was $47.1 \%$ [95\% confidence interval (Cl) 33.7-60.6\%]. Median PFS and OS was 9.2 months (95 \% Cl 7.6-10.8) and 22.5 months (95 \% Cl 19.4-25.9), respectively. Major adverse events (grade $3 / 4$ ) were neutropenia (9.3\%), thrombocytopenia (5.6\%), anorexia (18.5 \%), and sensory neuropathy $(16.7 \%)$.

Conclusion: These data suggested that SOX plus bevacizumab is effective and capable of being managed in metastatic CRC patients in our community clinical practice.

Keywords: Metastatic colorectal cancer, Chemotherapy, S-1, Prospective phase II study

\section{Introduction}

The combination chemotherapies, FOLFOX (5-fluorouracil [5-FU], leucovorin, and oxaliplatin) and FOLFIRI (5-FU, leucovorin, and irinotecan), have been the most common first-line metastatic colorectal cancer (CRC).

\footnotetext{
*Correspondence: k-shinozaki@hph.pref.hiroshima.jp

2 Division of Clinical Oncology, Hiroshima Prefectural Hospital, 1-5-54, Ujina-Kanda, Minami-ku, Hiroshima, Japan

Full list of author information is available at the end of the article
}

Targeted agents that enhance the effect of chemotherapy have been discovered, including bevacizumab (a humanized monoclonal antibody that targets vascular endothelial growth factor, a central regulator of angiogenesis), cetuximab and panitumumab (monoclonal antibodies directed against the epidermal growth factor receptor) (Hurwitz et al. 2004; Van Cutsem et al. 2009).

FOLFOX with bevacizumab is widely used in clinical practice as the first line treatment for metastatic CRC 
(Goldberg et al. 2004). However, this regimen is inconvenient owing to its requirement for continuous infusion via vascular access. To overcome this drawback, oral fluoropyrimidines, such as capecitabine, have been used as a substitute for infused leucovorin and fluorouracil. Recent data have demonstrated that capecitabine and oxaliplatin plus bevacizumab is non-inferior to FOLFOX plus bevacizumab regimen considering progression-free survival (PFS) of patients with metastatic CRC (Cassidy et al. 2008; Saltz et al. 2008).

Another oral fluorouracil, S-1, is a chemotherapy agent that consists of tegafur (a pro-drug of 5-FU) and two agents, gimeracil and oteracil, which decrease the rate of degradation of 5-FU anti-metabolite. S-1 could have advantages over capecitabine in terms of reducing the frequency of toxicities such as hand-foot syndrome (HFS), and several trials have shown the feasibility and efficacy of S-1/oxaliplatin (SOX) for metastatic CRC (Yamada et al. 2008; Zang et al. 2009). However, there is still a lack of sufficient evidence of efficacy and safety for this new treatment regimen to become a standard choice in clinical practice.

Accordingly, we conducted a multicenter clinical phase II trial across 12 institutions in Hiroshima, Japan (the Hiroshima Surgical study group of Clinical Oncology; HiSCO). We aimed to investigate the efficacy and safety of SOX plus bevacizumab, a promising alternative treatment for metastatic CRC.

\section{Patients and methods}

\section{Patient selection}

We undertook an open-label, non-randomized, multicenter clinical phase II trial in 12 institutions in Hiroshima, Japan. We enrolled individuals who met the following eligibility criteria: (1) histologically proven colorectal adenocarcinoma; (2) unresectable advanced/ metastatic CRC; (3) aged 20-80 years; (4) Eastern Cooperative Oncology Group performance status (PS) of 0 or 1 ; (5) presence of assessable lesions as confirmed on computed tomography or magnetic resonance imaging; (6) no previous chemotherapy or radiotherapy; (7) could take drugs orally; (8) adequate hematological, renal, and hepatic functions, as defined by a leucocyte count of $3-12 \times 10^{9} / \mathrm{L}$; neutrophil count of at least $2.0 \times 10^{9} / \mathrm{L}$; platelet count of at least $100 \times 10^{9} / \mathrm{L}$; hemoglobin level of at least $9.0 \mathrm{~g} / \mathrm{dL}$; total serum bilirubin concentration of no more than $1.5 \mathrm{mg} / \mathrm{dL}$; serum aspartate aminotransferase/serum alanine aminotransferase concentration of no more than $100 \mathrm{U} / \mathrm{L}$; serum creatinine concentration of no more than $1.2 \mathrm{mg} / \mathrm{dL}$; creatinine clearance $>60 \mathrm{~mL} /$ min; urinary protein score of no more than $1+$; and an international normalized ratio of no more than 1.5 and (9) estimated life expectancy of $>3$ months.
We excluded individuals if they had a history of serious allergies to any medications, active infections, serious concurrent disease, substantially impaired cardiac function, gastrointestinal ulcers or bleeding, sensory neuropathy, serious diarrhea, ascites or pleural effusion needing medication, brain metastases, a history of gastrointestinal perforation within the 6 months before enrollment, a history of thromboembolism or interstitial pneumonia, a history of surgery within the 28 days before enrollment, a blood coagulation disorder, were on anticoagulation medication, a history of hemoptysis, or had a primary lesion associated with a severe stricture that precluded passage of an endoscope. We also excluded individuals if they had previously or were presently receiving oxaliplatin-based regimens as adjuvant chemotherapy.

The current study was conducted in accordance with the declaration of Helsinki. All patients provided written informed consent after having been informed about the purpose and investigational nature of the study. The institutional review boards or ethics committees of each of the participating centers reviewed and approved the protocol. This study was registered in the UMIN Clinical Trial Registry as UMIN000004976.

\section{Treatment}

On day 1 of each 3-week cycle, patients assigned to receive SOX plus bevacizumab received a $7.5 \mathrm{mg} / \mathrm{kg}$ intravenous infusion of bevacizumab (for $30-90 \mathrm{~min}$ ), followed by an intravenous infusion of $130 \mathrm{mg} / \mathrm{m}^{2}$ oxaliplatin (for $2 \mathrm{~h}$ ). S-1 was taken orally twice daily from after dinner on day 1 to after breakfast on day 15 , followed by a 7-day break. The dose of S-1 was assigned according to body surface area: patients with a body surface area of less than $1.25 \mathrm{~m}^{2}$ received $80 \mathrm{mg} /$ day; those with a body surface area between $1.25 \mathrm{~m}^{2}$ and less than $1.5 \mathrm{~m}^{2}$ received $100 \mathrm{mg} /$ day; and those with a body surface area of at least $1.5 \mathrm{~m}^{2}$ received $120 \mathrm{mg} /$ day. Cycles were repeated for each patient until the criteria for withdrawal of the study treatment were met.

In view of the neurological toxicity of oxaliplatin, treatment could be skipped when patients had received at least $600 \mathrm{mg} / \mathrm{m}^{2}$ overall, even when no grade 3 toxic effects were recorded. If patients had grade 2 or higher proteinuria or grade 2 or higher bleeding before the scheduled starting day of each cycle, they received only SOX treatment; bevacizumab could be resumed in subsequent cycles if the treatment criteria were satisfied. The dose of cytotoxic drugs (oxaliplatin and S-1) was reduced by one level if the neutrophil count was less than $0.5 \times 10^{9} / \mathrm{L}$ at any time during a cycle, the neutrophil count was less than $1.0 \times 10^{9} / \mathrm{L}$ on the first day of a cycle, grade 3 or higher febrile neutropenia developed, or the platelet count was less than $50 \times 10^{9} / \mathrm{L}$. In the event of 
grade 3 or higher diarrhea, the dose of S-1 was reduced by one level. If the platelet count was between $50 \times 10^{9}$ and $75 \times 10^{9} / \mathrm{L}$ at any time during a cycle, or between $75 \times 10^{9}$ and $100 \times 10^{9} / \mathrm{L}$ on the first day of a cycle, the oxaliplatin dose was reduced by one level. S-1 was withheld when the neutrophil count was less than $1 \times 10^{9} / \mathrm{L}$; the platelet count was less than $75 \times 10^{9} / \mathrm{L}$; the serum creatinine concentration was more than $1.5 \mathrm{mg} / \mathrm{dL}$; suspected infection was diagnosed due to a fever of at least $38{ }^{\circ} \mathrm{C}$; or diarrhea, mucositis, or stomatitis of grade 2 or higher developed. S-1 was subsequently reinitiated when the neutrophil count was at least $1 \times 10^{9} / \mathrm{L}$; the platelet count was at least $75 \times 10^{9} / \mathrm{L}$; the serum creatinine concentration was less than $1.5 \mathrm{mg} / \mathrm{dL}$; no fever of $38{ }^{\circ} \mathrm{C}$ or higher suggesting infection was evident; and diarrhea, mucositis, and stomatitis were no higher than grade 1 .

\section{Endpoints}

The primary endpoint of this study was response rate (RR), and the secondary endpoints were PFS, overall survival (OS), and safety.

RR was calculated for patients who had measurable lesions using the Response Evaluation Criteria in Solid Tumor (RECIST; version 1.1) (Eisenhauer et al. 2009). $R R$ and disease control rate (DCR) were analyzed for the patients with target lesions. After initiation of study treatment, target and non-target lesions were assessed every 8 weeks in the same way as at baseline, using the same imaging conditions.

PFS was defined as the interval from the date of enrollment to the date on which progressive disease was first confirmed or the date of death from any cause, whichever came first. OS was defined as the interval from the date of enrollment to the date of death from any cause or last follow-up. Adverse events were graded according to the Common Terminology Criteria for Adverse Events (CTCAE; version 4.0).

We also evaluated the proportion of patients achieving disease control (a complete or partial response or stable disease), the proportion of patients having a curative resection, the time to treatment failure (TTF, interval from the date of enrolment to the date of a PFS event or withdrawal from the study for any reason), and adverse events.

\section{Statistical analysis}

All endpoints analyses except for the safety analysis were performed on the intent-to-treat set. The safety analysis included all treated patients who received at least one dose of the experimental drug. The required sample size was calculated to be at least 55 patients on the null hypothesis of a RR of $30 \%$ versus the alternative hypothesis of a RR of $50 \%$, with a power of $80 \%$, and a $95 \%$ significance level (one sided). Survival curves were estimated using the Kaplan-Meier method. All statistical analyses were performed using the IBM SPSS Statistic 20.0 software package.

\section{Results}

\section{Patient characteristics}

Between May 2011 and January 2014, 55 patients were enrolled in this study. Patient characteristics are listed in Table 1 . The mean age was 64 years. Forty-nine patients had a PS of 0 , and 6 had a PS of 1 . Of the 55 patients, 37 underwent primary tumor resection $(67.3 \%)$, and 12 underwent adjuvant chemotherapy (21.8\%). The number of organs with metastatic lesions was one in 29 patients $(52.7 \%)$ and two or more in 25 patients $(45.4 \%)$, and 2 patients had no assessable lesions (3.6\%). The median follow-up duration was 20.2 months (range 1.3-47.1).

\section{Efficacy}

One patient did not meet the eligibility criteria because of the absence of recurrent disease. Treatment was permanently stopped before the first tumor response evaluation for 4 patients owing to withdrawal of consent by 1 and owing to adverse events in the other 3 (grade 2 deep vein thrombosis in 1 patient, grade 3 anorexia in 1 patient, grade 4 neutropenia in 1 patient). In total, 472 treatment cycles were administered with a median of 7.5 cycles (range 0-34) per patient. S-1 was administrated in 472 cycles, oxaliplatin was administrated in 392 cycles, and bevacizumab was administrated in 423 cycles.

The tumor response data are listed in Table 2. RR was $47.1 \%$ [95 \% confidence interval (CI) 33.7-60.6\%], and therefore, the primary endpoint was achieved. DCR was $88.7 \%$ (95\% CI 80.1-97.2\%), and median TTF was 6.3 months (95\% CI 4.0-8.5 months). RR and DCR were analyzed for the patients with assessable lesions. A curative $\mathrm{R} 0$ resection was performed in 4 patients $(7.4 \%)$. A waterfall plot of the best overall response is demonstrated in Fig. 1.

Median PFS was 9.2 months (95 \% CI 7.5-10.9, Fig. 2), and median OS was 22.5 months (95 \% CI 19.425.9 months, Fig. 3).

\section{Safety}

The safety analysis included all treated patients who received at least one dose of the experimental drug $(\mathrm{n}=54)$. The adverse events are listed in Table 3. Major hematological adverse events (grade $3 / 4$ ) were neutropenia (9.3\%), thrombocytopenia (5.6\%), and leucopenia (5.6\%). Major non-hematological adverse events (grade $3 / 4$ ) were hypertension (22.2 \%), anorexia (18.5\%), and 
Table 1 Baseline patient characteristics $(n=55)$

\begin{tabular}{|c|c|}
\hline Parameter & Number of patients \\
\hline \multicolumn{2}{|l|}{$\operatorname{Sex}$} \\
\hline Male & 36 \\
\hline Female & 19 \\
\hline Age, years (range) & $64(21-79)$ \\
\hline \multicolumn{2}{|l|}{ Performance status } \\
\hline 0 & 49 \\
\hline 1 & 6 \\
\hline \multicolumn{2}{|l|}{ Primary site } \\
\hline Colon & 33 \\
\hline Rectosigmoid & 3 \\
\hline Rectum & 19 \\
\hline \multicolumn{2}{|l|}{ Tumor differentiation } \\
\hline well & 13 \\
\hline moderate & 36 \\
\hline poor & 2 \\
\hline others & 3 \\
\hline unknown & 1 \\
\hline \multicolumn{2}{|l|}{ Adjuvant chemotherapy } \\
\hline Yes (\%) & $12(21.8 \%)$ \\
\hline Uracil and tegafur plus leucovorin & 5 \\
\hline Capecitabine & 5 \\
\hline 5-FU/LV & 1 \\
\hline Uracil and tegafur & 1 \\
\hline Primary tumor resection (\%) & $37(67.3 \%)$ \\
\hline \multicolumn{2}{|l|}{ Assessable lesion } \\
\hline No & 2 \\
\hline Yes & 53 \\
\hline \multicolumn{2}{|l|}{ Metastatic sites } \\
\hline Liver & 32 \\
\hline Lung & 23 \\
\hline Lymph node metastases & 13 \\
\hline Peritoneal dissemination & 4 \\
\hline other metastases & 11 \\
\hline \multicolumn{2}{|l|}{ Number of metastatic sites } \\
\hline 1 & 29 \\
\hline 2 & 17 \\
\hline$\geq 3$ & 9 \\
\hline Liver limited disease (\%) & $14(25.4 \%)$ \\
\hline CEA (median, range) & $21.2(2.3-8577.6)$ \\
\hline CA19-9 (median, range) & $38.8(0.7-328,230)$ \\
\hline
\end{tabular}

5-FU 5-fluorouracil, LV leucovorin, CEA carcinoembryonic antigen, CA19-9 carbohydrate antigen 19-9

sensory neuropathy (16.7\%). The toxic effects were capable of being managed; however, attention to the occurrence of anorexia was needed.

The median relative dose intensities (RDIs; ratio of dose received to dose planned) are listed in Table 4 . The median RDI of oxaliplatin, S-1, and bevacizumab was 84 , 88 , and $90 \%$, respectively.
Table 2 Treatment outcomes

\begin{tabular}{|c|c|c|}
\hline Outcomes & $\begin{array}{l}\text { No. of patients } \\
(n=53)^{\mathrm{a}}\end{array}$ & $\begin{array}{l}95 \% \text { confidence } \\
\text { interval }\end{array}$ \\
\hline \multicolumn{3}{|l|}{ Response } \\
\hline Complete response & 1 & \\
\hline Partial response & 24 & \\
\hline Stable disease & 22 & \\
\hline Progressive disease & 2 & \\
\hline Not evaluable & 4 & \\
\hline Response rate (\%) & $25(47.1 \%)$ & $33.7-60.6$ \\
\hline Disease control rate (\%) & 47 (88.7 \%) & $80.1-97.2$ \\
\hline
\end{tabular}

a Response rate and disease control rate were analyzed for the patients with assessable lesion

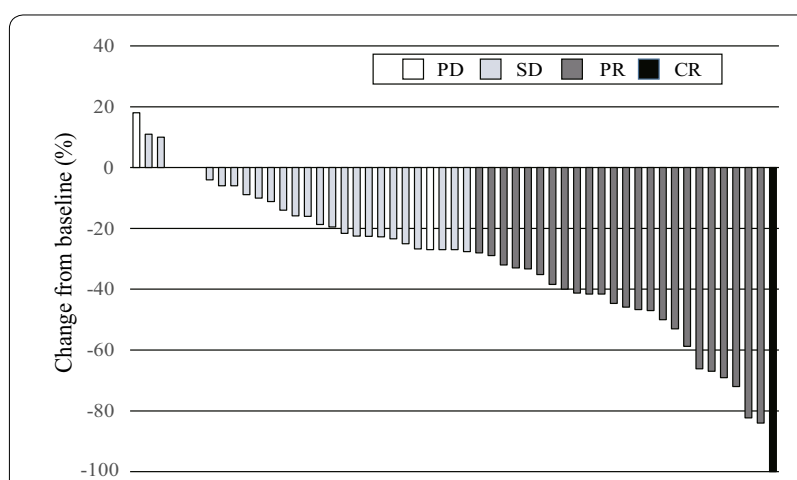

Fig. 1 Waterfall plot analysis of the best overall response in the intention to treat set. $C R$ complete response; $P R$ partial response; SD stable disease; $P D$ progressive disease

\section{Discussion}

We herein demonstrated that SOX plus bevacizumab is effective and capable of being managed in metastatic CRC patients in our community clinical practice.

The combination of chemotherapeutic regimens and the development of molecular targeted agents lead to improved survival in metastatic CRC. In order to develop new chemotherapeutic regimens, it is important to consider not only the survival benefit but also the maintenance of the quality of life of patients. S-1 is an effective derivative that combines tegafur with two modulators of 5-FU metabolism, gimeracil, a reversible inhibitor of dihydropyrimidine dehydrogenase, and oteracil in a molar ratio of 1:0.4:1. Gimeracil maintains a high fluorouracil concentration in the blood for a long time, and oteracil inhibits the conversion of 5-FU to active metabolites in the gastrointestinal tract, resulting in a reduction in gastrointestinal toxicity (Kato et al. 2001; Shirasaka 2009). S-1 was originally approved for the treatment of gastric cancer in Japan in 1999, and subsequently gained Japanese approval for CRC in 2003. More recently, there 


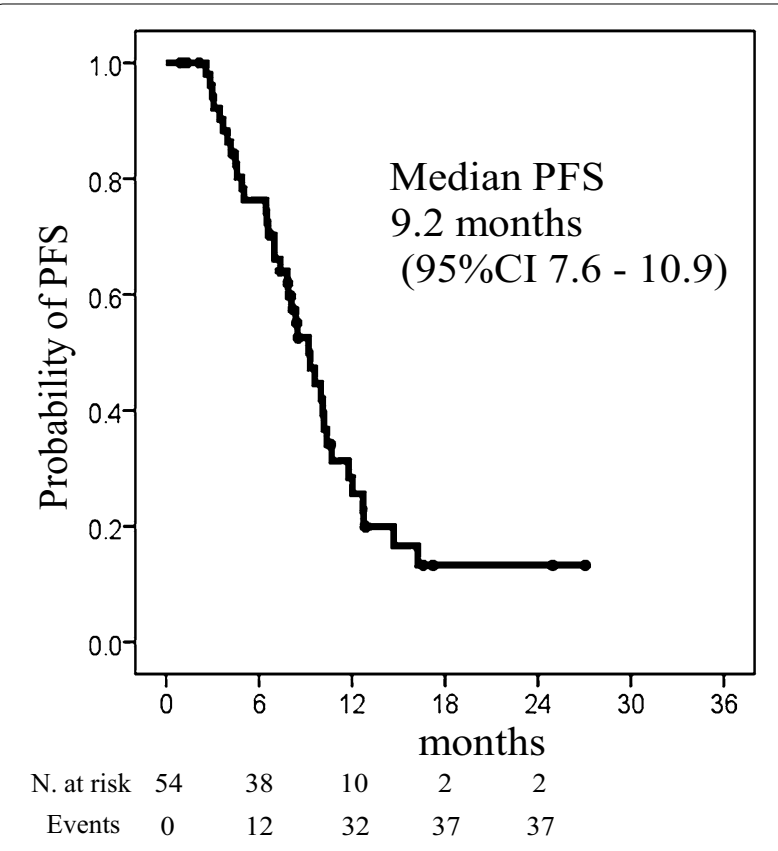

Fig. 2 Kaplan-Meier curves for progression-free survival (PFS); the median PFS was 9.2 months (95\% confidence interval: 7.6-10.8) N. at risk, number at risk

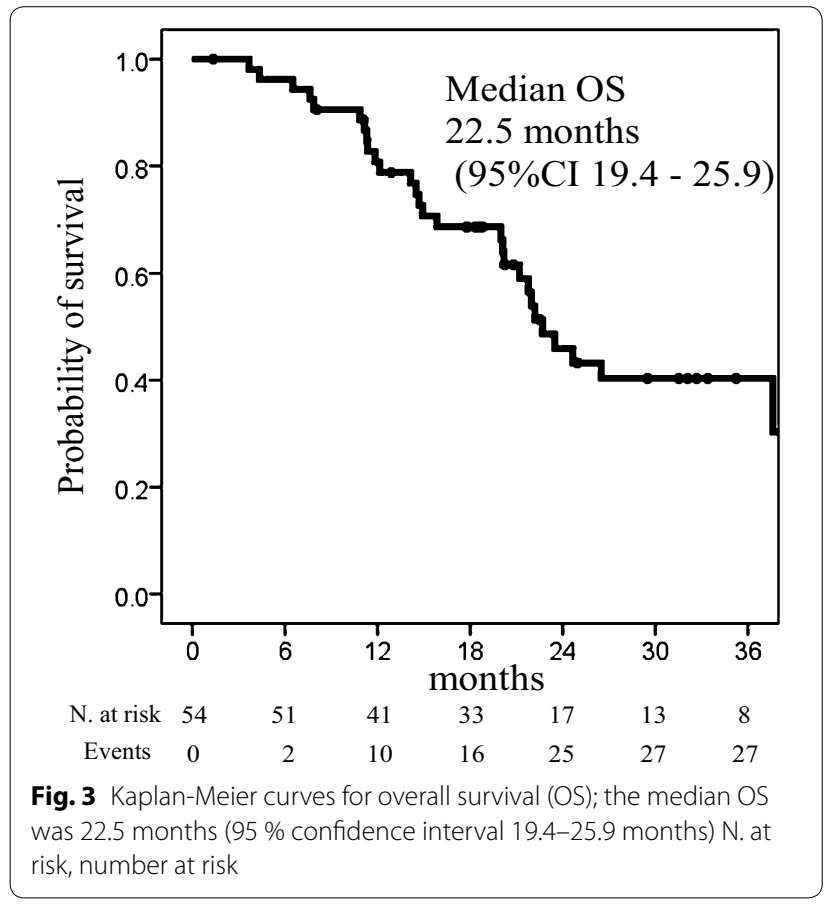

has been a shifting paradigm in cancer care towards oral chemotherapeutics. In the view of the convenience oral dosing offers to both patients and physicians, S-1 was gradually accepted as an alternative therapy to infused FU, similar to capecitabine (Muro et al. 2010; Yoshida et al. 2014). S-1 has the potential to improve accessibility to chemotherapy and decrease serious patient toxicities such as HFS. Placement of an ambulatory infusion pump is not absolutely necessary for patients treated with SOX plus bevacizumab. In our study, $33.3 \%$ of the patients $(\mathrm{n}=18)$ did not need an implanted port placement. Additionally, patients who received SOX plus bevacizumab returned to the hospital only once every 3 weeks. Thus, this new regimen is a promising treatment option to replace FOLFOX6 plus bevacizumab regimen, particularly for maintaining the quality of life of the patients.

In the phase I/II study of SOX regimen as the first-line treatment for metastatic CRC in Japan, Yamada et al. showed a RR of $50 \%$ and a median PFS of 6.4 months (Yamada et al. 2008). In the phase II study of SOX regimen in Korea, Zang et al. (2009) showed a RR of $54 \%$ and a median PFS of 8.5 months. A phase III study in South Korea showed that SOX is non-inferior to capecitabine plus oxaliplatin, with a RR of $47 \%$ and a median PFS of 8.5 months (Hong et al. 2012). Based on the results of these previous studies, we hypothesized the expected and threshold RRs of 50 and $30 \%$, respectively, used to design our study.

More recently, SOFT trial demonstrated the non-inferiority of SOX plus bevacizumab to MFOLFOX6 plus bevacizumab, with a RR of $61.5 \%$ and a median PFS of 10.2 months (Yamada et al. 2013). In the present study, the RR was $47.1 \%$ (95 \% CI 33.7-60.6\%), achieving the primary endpoint, and the median PFS was 9.2 months (95 \% CI 7.6-10.8 months, Fig. 1). Recent clinical trials have reported the median OS to be more than 30 months (Yamada et al. 2013; Schwartzberg et al. 2014). In this study, the median OS was 22.5 months (95\% CI 19.425.9 months). Additional investigation into the OS in the future is necessary, because our median follow-up duration was only 20.2 months.

The toxicity profile of SOX plus bevacizumab is known to be different from that of mFOLFOX6 and XELOX (capecitabine/oxaliplatin). In the present study, the frequency of severe (grade 3/4) hematological toxicities including thrombocytopenia were low; however, the occurrence of anorexia was high (18.5\%). HFS was rarely observed in the present study. The occurrence of sensory neuropathy was $16.7 \%$, which was almost equivalent to that occurring after the administration of FOLFOX6 or XELOX. The incidence of serial complications related to bevacizumab, such as venous thrombosis, gastrointestinal perforation, and bleeding complications, was extremely low. The occurrence of hypertension was high (grade 3/4, 22.2\%); therefore, medical management was needed. Generally, adverse events were capable of being managed in the present study; however, attention to the occurrence of anorexia was necessary. 
Table 3 Relative dose intensity

\begin{tabular}{llll}
\hline Drugs & Cycles & Median (\%) & Range (\%) \\
\hline S-1 & 472 & 84 & $23-100$ \\
Oxaliplatin & 392 & 88 & $52-100$ \\
Bevacizumab & 423 & 90 & $31-100$ \\
\hline
\end{tabular}

In the present study, the median RDI of oxaliplatin, S-1, and bevacizumab was 88,84 , and $90 \%$, respectively. In the phase I/II study of the SOX regimen in Japan, the median RDI of oxaliplatin and S-1 was 82.8 and $74.6 \%$, respectively (Yamada et al. 2008). In the phase II study of the SOX regimen in Korea, the median RDI of oxaliplatin and S-1 was 82 and $82 \%$, respectively (Zang et al. 2009). In a phase III study in South Korea, the median RDI of oxaliplatin and S-1 was 88 and $93 \%$, respectively (Hong et al. 2012). In SOFT trial, the median RDI of oxaliplatin, S-1, and bevacizumab was 75.5, 79.9, and $88.5 \%$, respectively (Yamada et al. 2013). These results suggest an almost equivalent RDI of the present study to those in previous studies.

Table 4 Safety analysis $(\mathbf{n}=\mathbf{5 4})$

\begin{tabular}{|c|c|c|}
\hline & $\begin{array}{l}\text { Any grade } \\
\text { n (\%) }\end{array}$ & $\begin{array}{l}\text { > Grade3 } \\
\text { n (\%) }\end{array}$ \\
\hline \multicolumn{3}{|l|}{ Haematological } \\
\hline Leucopenia & $30(55.6)$ & $3(5.6)$ \\
\hline Neutropenia & $30(55.6)$ & $5(9.3)$ \\
\hline Thrombocytopenia & $35(61.1)$ & $3(5.6)$ \\
\hline $\begin{array}{l}\text { Increased aspartate aminotransferase or alanine } \\
\text { aminotransferase concentration }\end{array}$ & $33(61.1)$ & $0(0)$ \\
\hline Increased creatinine concentration & $8(14.8)$ & $0(0)$ \\
\hline Proteinurea & $20(37)$ & $0(0)$ \\
\hline \multicolumn{3}{|l|}{ Non-haematological } \\
\hline Mucositis or stomatitis & $16(29.6)$ & $1(1.9)$ \\
\hline Anorexia & $38(70.4)$ & $10(18.5)$ \\
\hline Nausea & $16(29.6)$ & $3(5.6)$ \\
\hline Vomiting & $10(18.5)$ & $0(0)$ \\
\hline Diarrhoea & $16(29.6)$ & $0(0)$ \\
\hline Rash or desquamation & $11(20.4)$ & $0(0)$ \\
\hline Hyperpigmentation & $14(25.9)$ & $0(0)$ \\
\hline Fatigue & $36(68.7)$ & $1(1.9)$ \\
\hline Sensory neuropathy & $37(68.5)$ & $9(16.7)$ \\
\hline Hypertension & $46(85.2)$ & $12(22.2)$ \\
\hline Alopecia & $1(1.9)$ & $0(0)$ \\
\hline Hand-foot syndrome & $8(14.8)$ & $0(0)$ \\
\hline Gastroinetestinal obstrucion & $4(7.4)$ & $3(5.6)$ \\
\hline Gastrointestinal perforation & $0(0)$ & $0(0)$ \\
\hline Fever & $4(7.4)$ & $1(1.9)$ \\
\hline Thrombosis, thrombus, or embolism & $2(3.8)$ & $1(1.9)$ \\
\hline Bleeding complication & $5(9.2)$ & $1(1.8)$ \\
\hline
\end{tabular}

SOFT trial already showed the non-inferiority of SOX plus bevacizumab to mFOLFOX6 plus bevacizumab in a full analysis set without patients with peritoneal disseminations (Yamada et al. 2013). The new finding of our present study was that the primary endpoint was achieved in an intention to treat set including patients with peritoneal dissemination (four patients). The efficacy and safety of the SOX plus bevacizumab regimen was also demonstrated in this prospective multicenter phase II trial conducted in 12 institutions that play a major role in regional medicine in Hiroshima, Japan.

In conclusion, the SOX plus bevacizumab regimen is effective and capable of being managed in patients with advanced/metastatic CRC in our community clinical practice, and is an option for treatment to replace the FOLFOX plus bevacizumab regimen.

\section{Authors' contributions}

KS designed this study. MS and JT analyzed and interpretated the data. TH, MY, MK, DS, YI, TO, SK, YS, MA, MT, and MY participated in this clinical study and treated the patients. MS and KS wrote the paper. $\mathrm{HO}$ is the chief clinical investigator. All authors read and approved the final manuscript.

\section{Author details \\ ${ }^{1}$ Department of Gastroenterological and Transplant Surgery, Applied Life Sciences, Institute of Biomedical and Health Sciences, Hiroshima University, Hiroshima, Japan. ${ }^{2}$ Division of Clinical Oncology, Hiroshima Prefectural Hospital, 1-5-54, Ujina-Kanda, Minami-ku, Hiroshima, Japan. ${ }^{3}$ Department of Surgery, Hiroshima City Asa Hospital, Hiroshima, Japan. ${ }^{4}$ Department of Surgery, National Hospital Organization Higashihiroshima Medical Center, Higashihiroshima, Japan. ${ }^{5}$ Department of Surgery, Onomichi General Hos- pital, Onomichi, Japan. ${ }^{6}$ Department of Surgery, Chugoku Rosai Hospital, Kure, Japan. ${ }^{7}$ Department of Surgery, Hiroshima General Hospital of West Japan Railway Company, Hiroshima, Japan. ${ }^{8}$ Department of Surgery, Yoshida General Hospital, Akitakata, Japan. ${ }^{9}$ Department of Surgery, National Hospital Organization Kure Medical Center, Kure, Japan. ${ }^{10}$ Department of Surgery, Chuden Hospital, Hiroshima, Japan. ${ }^{11}$ Department of Surgery, National Hospital Organization Hiroshima-nishi Medical Center, Ohtake, Japan. ${ }^{12}$ Department of Surgery, Kure City Medical Association Hospital, Kure, Japan. ${ }^{13}$ Department of Epidemiology, Infectious Disease Control and Prevention, Institute of Biomedical and Health Sciences, Hiroshima University, Hiroshima, Japan.}

\section{Acknowledgements}

We thank all patients who participated in this trial and their families. The following departments and hospitals participated in the trial: Department of Gastroenterological and Transplant Surgery, Applied Life Sciences, Institute of Biomedical \& Health Sciences, Hiroshima University, Division of Clinical Oncology, Hiroshima Prefectural Hospital, Department of Surgery, Hiroshima City Asa Hospital, Department of Surgery, National Hospital Organization Higashihiroshima Medical Center, Department of Surgery, Onomichi General Hospital, Department of Surgery, Chugoku Rosai Hospital, Department of Surgery, Hiroshima General Hospital of West Japan Railway Company, Department of Surgery, Yoshida General Hospital, Department of Surgery, National Hospital Organization Kure Medical Center, Department of Surgery, Chuden Hospital, Department of Surgery, Hiroshima-nishi Medical Center, Department of Surgery, and Kure City Medical Association Hospital. We also thank Ms. Sanae Asano from the HiSCO (Hiroshima, Japan) for her excellent secretarial assistance.

\section{Competing interests}

The authors declare that they have no competing interests.

Received: 4 January 2016 Accepted: 7 October 2016

Published online: 18 October 2016 


\section{References}

Cassidy J, Clarke S, Diaz-Rubio E et al (2008) Randomized phase III study of capecitabine plus oxaliplatin compared with fluorouracil/folinic acid plus oxaliplatin as first-line therapy for metastatic colorectal cancer. J Clin Oncol 26:2006-2012

Eisenhauer EA, Therasse P, Bogaerts J et al (2009) New response evaluation criteria in solid tumours: revised RECIST guideline (version 1.1). Eur J Cancer 45:228-247

Goldberg RM, Sargent DJ, Morton RF et al (2004) A randomized controlled trial of fluorouracil plus leucovorin, irinotecan, and oxaliplatin combinations in patients with previously untreated metastatic colorectal cancer. J Clin Oncol 22:23-30

Hong YS, Park YS, Lim HY et al (2012) S-1 plus oxaliplatin versus capecitabine plus oxaliplatin for first-line treatment of patients with metastatic colorectal cancer: a randomised, non-inferiority phase 3 trial. Lancet Oncol 13:1125-1132

Hurwitz H, Fehrenbacher L, Novotny W et al (2004) Bevacizumab plus irinotecan, fluorouracil, and leucovorin for metastatic colorectal cancer. N Engl J Med 350:2335-2342

Kato T, Shimamoto Y, Uchida J et al (2001) Possible regulation of 5-fluorouracilinduced neuro- and oral toxicities by two biochemical modulators consisting of S-1, a new oral formulation of 5-fluorouracil. Anticancer Res 21:1705-1712

Muro K, Boku N, Shimada Y et al (2010) Irinotecan plus S-1 (IRIS) versus fluorouracil and folinic acid plus irinotecan (FOLFIRI) as second-line chemotherapy for metastatic colorectal cancer: a randomised phase 2/3 non-inferiority study (FIRIS study). Lancet Oncol 11:853-860
Saltz LB, Clarke S, Diaz-Rubio E et al (2008) Bevacizumab in combination with oxaliplatin-based chemotherapy as first-line therapy in metastatic colorectal cancer: a randomized phase III study. J Clin Oncol 26:2013-2019 Schwartzberg LS, Rivera F, Karthaus M et al (2014) PEAK: a randomized, multicenter phase II study of panitumumab plus modified fluorouracil, leucovorin, and oxaliplatin (mFOLFOX6) or bevacizumab plus mFOLFOX6 in patients with previously untreated, unresectable, wild-type KRAS exon 2 metastatic colorectal cancer. J Clin Oncol 32:2240-2247

Shirasaka T (2009) Development history and concept of an oral anticancer agent S-1 (TS-1): its clinical usefulness and future vistas. Jpn J Clin Oncol 39:2-15

Van Cutsem E, Kohne CH, Hitre E et al (2009) Cetuximab and chemotherapy as initial treatment for metastatic colorectal cancer. N Engl J Med 360:1408-1417

Yamada Y, Tahara M, Miya T et al (2008) Phase I/ll study of oxaliplatin with oral S-1 as first-line therapy for patients with metastatic colorectal cancer. Br J Cancer 98:1034-1038

Yamada Y, Takahari D, Matsumoto H et al (2013) Leucovorin, fluorouracil, and oxaliplatin plus bevacizumab versus S-1 and oxaliplatin plus bevacizumab in patients with metastatic colorectal cancer (SOFT): an open-label, non-inferiority, randomised phase 3 trial. Lancet Oncol 14:1278-1286

Yoshida M, Ishiguro M, Ikejiri K et al (2014) S-1 as adjuvant chemotherapy for stage III colon cancer: a randomized phase III study (ACTS-CC trial). Ann Oncol 25:1743-1749

Zang DY, Lee BH, Park HC et al (2009) Phase II study with oxaliplatin and S-1 for patients with metastatic colorectal cancer. Ann Oncol 20:892-896

\section{Submit your manuscript to a SpringerOpen ${ }^{\circ}$ journal and benefit from:}

- Convenient online submission

- Rigorous peer review

- Immediate publication on acceptance

- Open access: articles freely available online

- High visibility within the field

- Retaining the copyright to your article 